\title{
Lung ultrasound score-based perioperative
} assessment of pressure-controlled ventilationvolume guaranteed or volume-controlled ventilation in geriatrics: a prospective randomized
controlled trial

This article was published in the following Dove Press journal: Clinical Interventions in Aging

Young Sung Kim, Young Ju Won, Dong Kyu Lee, Byung Gun Lim, Heezoo Kim, II Ok Lee, Jin Hee Yun, Myoung Hoon Kong

Department of Anesthesiology and Pain Medicine, Korea University Guro Hospital, Korea University College of Medicine, Guro-gu, Seoul, Republic of Korea

\section{Video abstract}

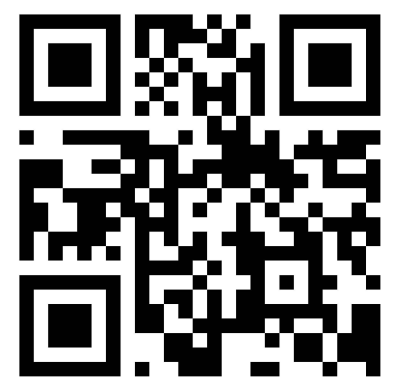

Point your SmartPhone at the code above. If you have a QR code reader the video abstract will appear. Or use: https://www.youtube.com/watch?v=94P0Mcek0jl

Correspondence: Young Ju Won Department of Anaesthesiology and Pain Medicine, Korea University Guro

Hospital, Gurodong-ro 148, Guro-gu, Seoul 08308 , Republic of Korea

Tel +82 $22626 \quad 1437$

Fax +82226261438

Email moma2@naver.com
Purpose: Recent studies have shown the potential benefits of pressure-controlled ventilation-volume guaranteed (PCV-VG) compared to volume-controlled ventilation (VCV), but the results were not impressive. We assessed the effects of PCV-VG versus VCV in elderly patients by using lung ultrasound score (LUS).

Patients and methods: Elderly patients (aged 65-90 years) scheduled for hip joint surgery were randomly assigned to either the PCV-VG or VCV group during general anesthesia. LUS and mechanical ventilator parameters were evaluated before induction, 30 mins after a semi-lateral position change, during supine repositioning before awakening, and 15 mins after arrival to the post-anesthesia care unit (PACU). Pulmonary function tests were performed before and after surgery. Other recovery indicators were also assessed in the PACU. Results: A total of 76 patients (40 for PCV-VG and 36 for VCV) were included this study. Demographic data showed no significant difference between the two groups. In both groups, LUSs before induction were significantly lower than those at other time points. LUSs of the VCV group were significantly increased during perioperative periods compared with the PCV-VG group ( $p=0.049$ ). Visualized LUS modeling suggested an intuitive difference in the two groups and unequal distribution in lung aeration. Higher dynamic compliance and lower inspiratory peak pressure were observed in the PVC-VG group compared to the VCV group (33.54 vs 27.36, $p<0.001 ; 18.93$ vs $21.19, p<0.001$, respectively). Postoperative forced vital capacity of the VCV group was lower than that of PCV-VG group, but this result was not significant (2.06 vs 1.79 , respectively; $p=0.091$ ). The other respiratory data are comparable between the two groups.

Conclusion: The PCV-VG group showed better LUS compared with the VCV group. Moreover, LUS modeling in both groups suggests non-homogeneous and positional change in lung aerations during surgery.

Clinical trial registration: This study was registered at the UMIN clinical trials registry (unique trial number: UMIN000029355; registration number: R000033510)

Keywords: geriatrics, hip joint surgery, mechanical ventilation, lung physiology, ultrasonography

\section{Introduction}

The number of elderly people is increasing, and the cost involved in the treatment of hip fractures has become a social burden. ${ }^{1}$ Moreover, the pulmonary changes that occur 
with aging are associated with a number of physiological, morphological, and histological changes that reduce respiratory function reserves. ${ }^{2}$ Postoperative respiratory complications also significantly increase according to patients' increasing age, ${ }^{3}$ and the high incidence of lung complications including pneumonia and mortality ${ }^{4}$ presents a challenge for clinicians. For anesthesiologists, it is important to prevent and protect patients from respiratory complications ${ }^{4,5}$ in the perioperative periods. When a patient's spontaneous breathing ceases during general anesthesia, it results in unbalanced ventilation of the alveoli as a result of the patient's respiration; in such cases, the patients are placed on positive pressure mechanical ventilation. ${ }^{6}$ In this context, it is natural for an anesthesiologist to evaluate the ventilation mode during surgery.

Volume-controlled ventilation (VCV) has been the most common mode of mechanical ventilation used during general anesthesia. Although VCV may provide the relatively precise minute volume of ventilation, it can lead to high peak inspiratory pressure in the alveoli that may result in barotrauma to the lungs. Pressure-controlled ventilationvolume guaranteed (PCV-VG) is a mechanical ventilation mode that guarantees tidal volume with the lowest possible pressure using decelerating flow. ${ }^{7}$ PCV-VG is known to improve arterial oxygenation and has a rapidly decelerating flow pattern. Lower peak pressure $\left(\mathrm{P}_{\text {peak }}\right)$ and higher compliance may be beneficial in reducing barotrauma. ${ }^{7}$ However, despite the many reports of the effects of mechanical ventilation during surgery to manage respiratory changes in elderly patients, the potential benefits of PCVVG compared to VCV are controversial. ${ }^{8-10}$ To better understand the differences between the two groups, we tried to identify a new evaluation index. We thought that lung ultrasound scores (LUS) can be used to evaluate patients' respiratory status quickly and appropriately during surgery. In this study, we demonstrate the effects of PCVVG and VCV in elderly patients by using transthoracic LUS, pulmonary function tests (PFT), arterial blood gas analysis (ABGA), airway pressure, and hemodynamic data. We hypothesized that PCV-VG may result in better LUS by reducing atelectasis in the dependent area of the lung and minimizing respiratory deterioration during the postoperative period in elderly patients.

\section{Methods}

This study was a single-center prospective randomized controlled trial performed at Korea University Guro Hospital, Seoul, South Korea, from October 2017 to October 2018.
After obtaining approval from the Korea University Guro Hospital Institutional Review Board(IRB \#2017GR0820), written informed consent was obtained from all subjects participating in the trial. The trial was registered prior to patient enrollment in the UMIN clinical trials registry (unique trial number: UMIN000029355; registration number: R000033510, principal investigator: Young ju Won, Date of registration: 28, Sep, 2017). This study was presented in accordance with the Consolidated Standards of Reporting Trials (CONSORT) guidelines. All patients were recruited from the Department of Orthopedic Surgery, Korea University Guro Hospital by the research staff. Patients were enrolled in the study after the registration of UMIN clinical trials registry at the hospital the day before surgery. After providing an explanation of the trial, written informed consent was obtained from all participants. The original data have been registered at the UMIN-ICDR (Registration No. UMIN000029355). This study was conducted in accordance with the Declaration of Helsinki.

Patients were aged 65 to 90 years, had an American Society of Anesthesiologists physical status I to III, had a BMI $\leq 31 \mathrm{~kg} / \mathrm{m}^{2}$, had no history of acute respiratory disease, and were scheduled for hip joint surgery under general anesthesia. Patients with mental retardation or severe cognitive impairment, history of a previous intrathoracic procedure, or a cardiopulmonary compromised status were excluded from the study. Patients who refused to participate in the study were also excluded. Demographic data including age, sex, weight, height, and the Charlson comorbidity index were collected in the patients (Table 1). Patients were randomly allocated to the PCV-VG group or the VCV group based on the ventilator mode chosen, and they were unaware of the assigned group. A single investigator was responsible for the group assignment of patients. Randomization was achieved using a web-based computer-generated list (www.randomization.com). The numbers were kept in opaque, sealed envelopes that were opened

Table I Demographic data

\begin{tabular}{|l|l|l|}
\hline & $\begin{array}{l}\text { Group PCV-VG } \\
(\mathbf{n = 4 0 )}\end{array}$ & $\begin{array}{l}\text { Group VCV } \\
(\mathbf{n}=36)\end{array}$ \\
\hline Age (years) & $77.35 \pm 5.99$ & $78.97 \pm 5.95$ \\
Sex (M/F) & $11 / 29$ & $8 / 28$ \\
Weight (kg) & $57.87 \pm 8.37$ & $54.49 \pm 10.16$ \\
Height (cm) & $156.04 \pm 7.73$ & $155.93 \pm 8.66$ \\
Charlson comorbidity Index & $11 / 14 / 5 / 8$ & $7 / 16 / 3 / 9$ \\
$(0 / / / 2 / \geq 3)$ & & \\
\hline
\end{tabular}

Note: Values are either the mean \pm SD or the number of patients. Abbreviations: PCV-VG, pressure-controlled ventilation-volume guaranteed; VCV, volume-controlled ventilation. 
in the operating room by an independent anesthesiologist not involved in the study.

\section{Anesthetic protocol}

Non-invasive blood pressure measurement, electrocardiogram, pulse oximetry (CARESCAPE monitor B650, GE healthcare), and state entropy (GE Healthcare, Helsinki, Finland) were all monitored in each patient. We also applied neuromuscular blockade monitoring device (TOF-Watch SX; Organon Ltd, Ireland) to monitor train-of-four (TOF, frequency $2 \mathrm{~Hz}$, current $50 \mathrm{~mA}$, interval $15 \mathrm{~s}$ ). The baseline values for mean blood pressure, heart rate, surgical pleth index, and state entropy were recorded before anesthesia induction. All anesthetic procedures were carried out by two independent anesthesiologists not involved in the study. One blinded independent anesthesiologist performed the induction and maintenance of anesthesia, administered the study drug according to the study protocol, and recorded the values. The other blinded independent anesthesiologist assessed the extubation time and postoperative pain during the emergence and recovery phases.

Anesthesia induction was achieved using propofol $2 \mathrm{mg} /$ $\mathrm{kg}$, remifentanil, rocuronium $0.6 \mathrm{mg} / \mathrm{kg}$, and mask ventilation with desflurane and oxygen $8 \mathrm{~L} / \mathrm{min}$ for $2 \mathrm{~min} 30 \mathrm{sec}$, followed by intubation (7.5 to 8.0 for male and 6.5 to 7.0 for female of sized Portex tracheal tube). Mechanical ventilation was maintained at a tidal volume of $8 \mathrm{~mL} / \mathrm{kg}, 1: 2$ of inspiration-to expiration ratio (I:E ratio) and ventilation frequency (10 to 14 per minute of respiratory rate) was adjusted to maintain end-tidal carbon dioxide $\left(\mathrm{P}_{\mathrm{et}} \mathrm{CO}_{2}\right)$ at $30-35 \mathrm{mmHg}$ (GE Datex-Ohmeda Aestiva 3000; GE Healthcare, Wauwatosa, WI, USA). A pressure limit of peak inspiratory pressure was $40 \mathrm{mmHg}$. To obtain the plateau pressure value, $5 \%$ of inspiratory pause was applied in the VCV group. Routine positive end-expiratory pressure (PEEP) of $5 \mathrm{cmH}_{2} \mathrm{O}$ was applied. If the end expiratory pressure is not equal to $5 \mathrm{cmH}_{2} \mathrm{O}$ (set PEEP), which indicates auto-PEEP, we carefully inspected overall situation to detect errors including endotracheal tube (size, depth and kinking), inadequate ventilation, patient's malposition, fault in anesthetic circuit, water accumulation in the ventilator tube, long inspiratory times and bronchospasm. Anesthesia was maintained with desflurane inhalation in $50 \%$ air-oxygen mixture at $3.0 \mathrm{~L} / \mathrm{min}$ to achieve state entropy of 40-60, and also by continuously adjusted the remifentanil intravenous infusion for a mean arterial pressure of $65-95 \mathrm{mmHg}$ and a heart rate of $80-100$ beats per minute. For the proper neuromuscular blockade, neuromuscular function was monitored with an acceleromyograph, using the TOF-Watch $^{\circledR}$ SX (Organon Ireland Ltd, Schering-Plough
Corporation, Dublin, Ireland). Five milligrams of rocuronium was additionally administered at the reappearance of 2 counts from the train-of-four (T2) until a subcuticular suture was made. At the end of surgery, the administration of desflurane and remifentanil was stopped, fresh gas flow was increased to $8 \mathrm{~L} / \mathrm{min}$ of oxygen, and sugammadex $2 \mathrm{mg} / \mathrm{kg}$ was administered to reverse the neuromuscular blockade, after confirming a TOF count of 2. After the patient recovered spontaneous breathing and consciousness, extubation was performed and the patient was transferred to the post-anesthesia care unit (PACU).

In the PACU, the blinded independent anesthesiologist assessed the recovery time (time to reach a modified Aldrete score of 10), sedation scale (the Richmond Agitation and Sedation Scale; RASS), presence or absence of delirium (the Confusion Assessment Method for the Intensive Care Unit; CAM-ICU), verbal numeric rating scale (VNRS; 110) for pain every $10 \mathrm{~min}$ for $60 \mathrm{~min}$, cumulative consumption of fentanyl, and the occurrence of adverse events.

\section{Evaluation of peri-operative pulmonary status}

Preoperative PFT was performed the day prior to surgery by using the MicroLab apparatus (Micro Medical, Kent, UK) with the patients in the Fowler position (semi-upright, $60 \%$ head-up) with legs uncrossed. Postoperative PFT were performed in the same way, after patients' VNRS scores were less than 4, they had a modified Aldrete score of 10, and had no postoperative delirium at the time. The parameters used in the PFT include forced expiratory volume at timed intervals of 1.0 seconds (FEV1), forced vital capacity (FVC), and a FEV1/FVC ratio.

The primary endpoint of our study was to assess LUS. After arrival in the operating room, measurement of LUS and data collection were performed by the same two anesthetists, who had experience with more than 100 cases. LUS scans were performed by using a Sonosite Edge ultrasound system (Fuji Film, Bothell, WA, USA) and a 6-12 MHz linear probe. The default scan depth was set at $4.9 \mathrm{~cm}$ and if B-lines was suspected, we increased depth to rule out artifacts including Z-line which is known to fade out at a lesser depth. LUS examination was performed at four different time points for each patient: before induction (time point 1 [TP1]), 30 mins after a semi-lateral position change (TP2), during supine repositioning before awakening (TP3), and 15 mins after arrival to the post-anesthesia care unit (PACU) (TP4). Patients 
were scanned in the supine position following the LUS examination method described by Monastesse and colleagues. The thorax was divided by the anterior axillary line, the posterior axillary line, and a horizontal line beneath the nipple. Intercostal spaces of each twelve area were scanned and analyzed. Aeration loss was assessed by calculating the modified LUS. Two lung ultrasound examiners provided scores at each area after simultaneously examining the lung scan. The LUS of the thorax (0-36) was then calculated by summing the twelve individual quadrant scores, with higher scores indicating more severe aeration loss. LUS were evaluated when the patient was in the supine position before induction (TP1), 30 mins after a lateral position change (TP2), at the time of supine repositioning before awakening (TP3), and 15 mins after arrival to the PACU (TP4). ABGA were also performed at $\mathrm{TP} 1,2$, and 3. Respiratory parameters including tidal volume, respiratory rate, peak inspiratory pressure, plateau pressure, end expiratory pressure, $\mathrm{P}_{\mathrm{et}} \mathrm{CO}_{2}$, partial pressure of carbon dioxide $\left(\mathrm{P}_{\mathrm{a}} \mathrm{CO}_{2}\right)$, partial pressure of arterial oxygen $\left(\mathrm{PaO}_{2}\right)$, blood oxygen saturation, static and dynamic compliance $\left(\mathrm{C}_{\text {stat }}\right.$ and $\mathrm{C}_{\mathrm{dyn}}$, respectively), and alveolar dead space (DS) volume were evaluated at TP1, 2, and 3. $\mathrm{C}_{\text {stat }}(1)$ and $\mathrm{C}_{\mathrm{dyn}}(2)$ were calculated as:

$$
\begin{aligned}
\mathrm{C}_{\text {stat }}= & \text { tidal volume } /(\text { plateau pressure } \\
& \text { - positive end expiratory pressure })
\end{aligned}
$$

$$
\begin{aligned}
\mathrm{C}_{\mathrm{dyn}} & =\text { tidal volume } /(\text { peak inspiratory pressure } \\
& \text { - positive end expiratory pressure })
\end{aligned}
$$

The ratios of alveolar DS to tidal volume were calculated using Hardman and Aitkenhead Equation (3) as:

$$
\mathrm{DS}=1.14 \times\left(\mathrm{P}_{\mathrm{a}} \mathrm{CO}_{2}-\mathrm{P}_{\mathrm{et}} \mathrm{CO}_{2}\right) / \mathrm{P}_{\mathrm{a}} \mathrm{CO}_{2}-0.005
$$

\section{Statistical analysis}

A power analysis suggested that a minimum sample size of 40 patients for each group would be required with a significance level of $5 \%$ to achieve a power of $80 \%$. The power analysis was calculated from our preliminary data using a Independent $t$-test: the mean difference of the total LUS at the PACU between the PCV-VG (mean of 10.0 and common standard deviation [SD] of 4.9) and VCV group (mean of 13.1 and common SD of 4.8). To allow for an exclusion rate, the study population was prospectively set at 88 patients.

The analyzed data were tested for normality using the Kolmogorov-Smirnov test. Either a parametric or non- parametric analysis was performed depending upon the results of the Kolmogorov-Smirnov analysis. Data were expressed as the mean $\pm \mathrm{SD}$ and compared using independent $t$-tests or Mann-Whitney $\mathrm{U}$ tests for intergroup analysis. For repeated measurements including LUS, PFT, and respiratory parameters, paired $t$-tests were used in each group to comparing each respective time point to the initial time point. In addition, repeated measures analysis of variance (ANOVA) was used for analysis of group effects on LUS. When the sphericity condition of data was not satisfied, the results from multivariate analyses were adopted. On the contrary, when the sphericity condition of data was satisfied, we adopted the results of tests showing within-subjects' effects. Categorical variables were compared using a chi-square test or Fisher's exact test as appropriate.

Statistical analyses were performed with SPSS 22 (IBM, Armonk, NY, USA, Statistical Package for the Social Science 22). A $p$-value of $<0.05$ was considered significant.

\section{Results}

Except for 12 patients who withdrew consent or failed screening, 76 patients were enrolled in this study (40 patients in the PCV-VG group and 36 patients in the VCV group) (Figure 1). Demographic data showed no significant differences between the two groups (Table 1). Perioperative outcomes including total anesthesia time; the amount of transfusion and colloid use; total fluid; blood loss; urine output; recovery profiles including VNRS, RASS, CAM-ICU; and opioid consumption were not different between the two groups (Table 2). In both groups, any significant adverse event including auto-PEEP was not observed during surgery. And no critical complications was reported during the whole study periods.

Peak inspiratory pressures are high in VCV group, and dynamic compliance are lower in VCV group compared to PCV-VG group, other respiratory parameters are comparable during surgery (Table 3 ). The numbers of patients with delirium were $7(17.5 \%)$ in the PCV-VG group and $3(8.3 \%)$ in the VCV group. Except for the patients with delirium, 33 patients $(82.5 \%)$ in the PCV-VG group and 33 patients (91.7\%) in the VCV group completed PFT. Postoperative FEV1 values (1.47 for the PCV-VG group; 1.35 for the VCV group; $p=0.226$ between the two groups) and FVC values (2.06 for the PCV-VG group; 1.79 for the VCV group; $p=0.091$ between the two groups) were significantly lower than the preoperative values in both groups $(\mathrm{FEV} 1=1.70, \mathrm{FVC}=2.36$, and $p<0.001$ for the PCV-VG 


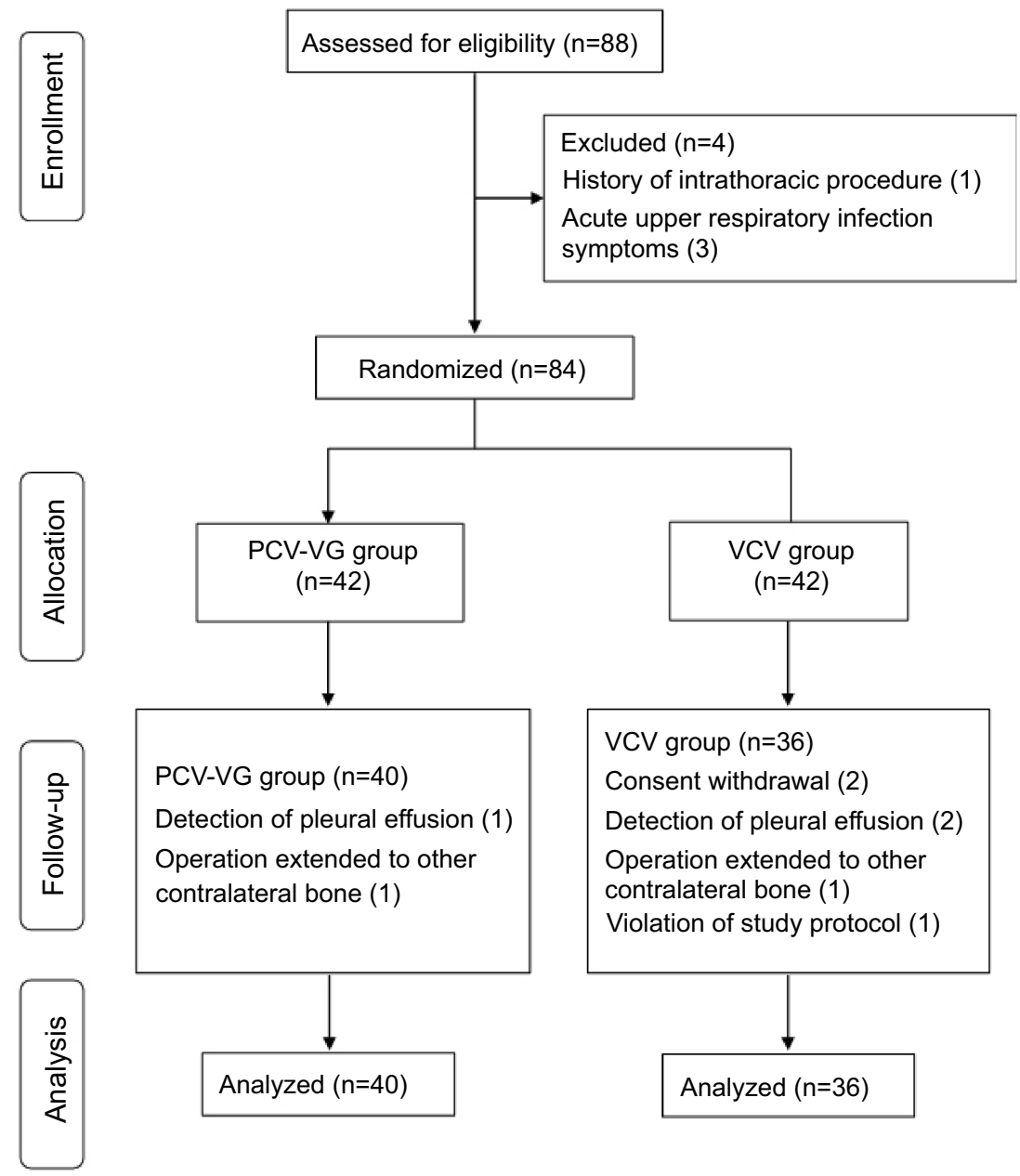

Figure I Flow diagram.

Abbreviations: PCV-VG, pressure-controlled ventilation-volume guaranteed; VCV, volume-controlled ventilation.

group; FEV1 $=1.66, \mathrm{FVC}=2.26$, and $p<0.001$ for the $\mathrm{VCV}$ group) (Table 2).

LUS at TP1 were lower than other time points in most cases, prominently in the VCV group. The changes of LUS of the total compartments, and the anterior and left compartments were significantly different between the two groups $(\mathrm{F}=2.745,4.178$, and 3.371; $p=0.049,0.007$, and 0.019, respectively). The changes to the LUS in the dependent lung compartments seemed to show greater fluctuations compared to those in nondependent compartments. (Figure 2)

Figure 3 shows a visual model of the LUS results. Compared to the PCV-VG group, the VCV group showed more dark-colored compartments (the darkness or brightness of the color corresponds to the LUS) in the perioperative and postoperative periods (Figure 3 ).

\section{Discussion}

In this study, we demonstrated favorable LUS results, higher dynamic compliance, and low inspiratory peak pressure in the PCV-VG compared to those in the VCV group. There have been several studies comparing pressurecontrolled ventilation (PCV) and VCV, whose findings have been consistent with ours, showing increased peak inspiratory pressure in VCV. Many studies have focused on situations that result in high peak inspiratory pressures, such as abdominal endoscopic or robot surgery, ${ }^{9,11,12}$ one-sided lung ventilation, ${ }^{13,14}$ or surgeries performed on obese patients. ${ }^{15-17}$ Jaju et $\mathrm{al}^{11}$ showed a lower $\mathrm{P}_{\mathrm{a}} \mathrm{CO}_{2}$ and $\mathrm{P}_{\mathrm{a}} \mathrm{CO}_{2}-\mathrm{EtCO}_{2}$ gradient, suggesting a better preserved ventilation-perfusion match in PCV. In the instance of the occurrence of pneumoperitoneum in the Trendelenburg position, a rise in central venous pressure was more significant when using VCV. For similar reasons, Lian et $\mathrm{al}^{9}$ suggest that PCV would be a better choice, even though the changes of DS during VCV or PCV were similar. Hosten et $\mathrm{al}^{18}$ reported a higher $\mathrm{PaO}_{2} /$ fraction of inspired oxygen $\left(\mathrm{FiO}_{2}\right)$ ratio at the end of surgery when using PCV. Messeha $^{16}$ also provided evidence for improved 
Table 2 Perioperative outcomes and recovery profiles

\begin{tabular}{|l|l|l|}
\hline & Group PCV-VG (n=40) & Group VCV (n=36) \\
\hline ANE time (min) & $163.25 \pm 54.86$ & $147.08 \pm 56.83$ \\
OP position (RLD/LLD) & $23 / 17$ & $16 / 20$ \\
Transfusion (Y/N) & $10 / 30$ & $7 / 29$ \\
- RBC (pack) & $0.22 \pm 0.50$ & $0.23 \pm 0.57$ \\
- FFP (pack) & $0.06 \pm 0.32$ & $0.09 \pm 0.37$ \\
Colloid use (Y/N) & $1 / 39$ & $1 / 35$ \\
Total fluid (mL) & $704.00 \pm 425.22$ & $624.44 \pm 356.63$ \\
Blood loss (mL) & $293.50 \pm 364.94$ & $248.33 \pm 317.95$ \\
Urine output (mL) & $340.63 \pm 277.68$ & $312.50 \pm 235.68$ \\
Fentanyl use in PACU (mcg) & $95.43 \pm 42.71$ & $101.67 \pm 35.05$ \\
VNRS in PACU at I hr & $3.48 \pm 1.92$ & $3.33 \pm 1.12$ \\
RASS in PACU at I hr & $0.00 \pm 0.72$ & $-0.03 \pm 0.45$ \\
CAM-ICU in PACU at I hr (positive/negative) & $7 / 33$ & $3 / 33$ \\
FEV (pre/postoperative) & $1.74 \pm 0.48 / 1.47 \pm 0.43^{\mathrm{a}}$ & $1.65 \pm 0.44 / 1.35 \pm 0.40^{\mathrm{a}}$ \\
FVC (pre/postoperative) & $2.44 \pm 0.68 / 2.06 \pm 0.65^{\mathrm{a}}$ & $2.26 \pm 0.61 / 1.79 \pm 0.61^{\mathrm{a}}$ \\
\hline
\end{tabular}

Notes: Values are mean $\pm S D$ or number of patients. ${ }^{a} p<0.05$ compared to the preoperative value.

Abbreviations: PCV-VG, pressure-controlled ventilation-volume guaranteed; VCV, volume-controlled ventilation; RLD, Right lateral decubitus; LLD, Left lateral decubitus; PACU, post-anesthesia care unit; VNRS, verbal numeric rating scale; RASS, the Richmond agitation and sedation scale; CAM-ICU, confusion assessment method for the ICU; $\mathrm{FEV}_{1}$, forced expiratory volume at timed intervals of 1.0 seconds; FVC, forced vital capacity.

Table 3 Changes in respiratory parameters during surgery

\begin{tabular}{|l|l|l|}
\hline & Group PCV-VG (n=40) & Group VCV (n=36) \\
\hline PIP - Preoperative & $17.98 \pm 2.27$ & $20.39 \pm 2.56^{\mathrm{a}}$ \\
- Decubitus position & $19.63 \pm 2.67$ & $22.33 \pm 2.58^{\mathrm{a}}$ \\
- Before awakening & $18.93 \pm 2.67$ & $21.19 \pm 2.75^{\mathrm{a}}$ \\
PP - Preoperative & $17.98 \pm 2.27$ & $18.06 \pm 2.33$ \\
- Decubitus position & $19.48 \pm 3.04$ & $19.92 \pm 2.12$ \\
- Before awakening & $18.77 \pm 2.89$ & $18.64 \pm 2.75$ \\
$\mathrm{P}_{\mathrm{a}} \mathrm{CO}_{2}$ - Preoperative & $36.38 \pm 4.43$ & $35.44 \pm 3.61$ \\
- Decubitus position & $34.73 \pm 3.35$ & $35.22 \pm 3.43$ \\
- Before awakening & $35.60 \pm 3.66$ & $35.56 \pm 3.88$ \\
$\mathrm{PaO}_{2}$ - Preoperative & $212.04 \pm 29.93$ & $202.78 \pm 37.39$ \\
- Decubitus position & $205.10 \pm 32.35$ & $194.81 \pm 42.96$ \\
- Before awakening & $202.80 \pm 33.13$ & $194.19 \pm 40.18$ \\
$\mathrm{C}_{\mathrm{dyn}}$ - Preoperative & $35.70 \pm 7.22$ & $28.95 \pm 5.64^{\mathrm{a}}$ \\
- Decubitus position & $31.99 \pm 7.00$ & $25.62 \pm 4.25^{\mathrm{a}}$ \\
- Before awakening & $33.54 \pm 7.23$ & $27.36 \pm 5.90^{\mathrm{a}}$ \\
$\mathrm{C}_{\text {stat }}$ - Preoperative & $35.70 \pm 7.22$ & $34.34 \pm 7.49$ \\
- Decubitus position & $33.48 \pm 13.00$ & $29.81 \pm 5.44$ \\
- Before awakening & $34.19 \pm 8.07$ & $33.00 \pm 8.75$ \\
DS - Preoperative & $0.15 \pm 0.07$ & $0.15 \pm 0.08$ \\
- Decubitus position & $0.16 \pm 0.08$ & $0.16 \pm 0.08$ \\
- Before awakening & $0.17 \pm 0.09$ & $0.19 \pm 0.09$ \\
\hline
\end{tabular}

Notes: Values are mean \pm SD. ${ }^{a} p<0.05$ compared to the PCV-VG group.

Abbreviations: PIP, peak inspiratory pressure; PP, plateau pressure; $\mathrm{P}_{\mathrm{a}} \mathrm{CO}_{2}$, partial pressure of carbon dioxide; $\mathrm{PaO}$, partial pressure of arterial oxygen; Cdyn, dynamic compliance; Cstat, static compliance; DS, alveolar dead space; PCV-VG, pressure-controlled ventilation-volume guaranteed; VCV, volume-controlled ventilation.

intraoperative oxygenation when switching from VCV to PCV. These benefits are seen not only in respiratory outcomes; other PCV advantages are also being reported.
Compared to the PCV group, the VCV group seemed to modulate pro-inflammatory factors such as interleukin (IL)$10,{ }^{19} \mathrm{IL}-6$, and tumor necrosis factor alpha $(\mathrm{TNF} \alpha) .{ }^{14}$ 

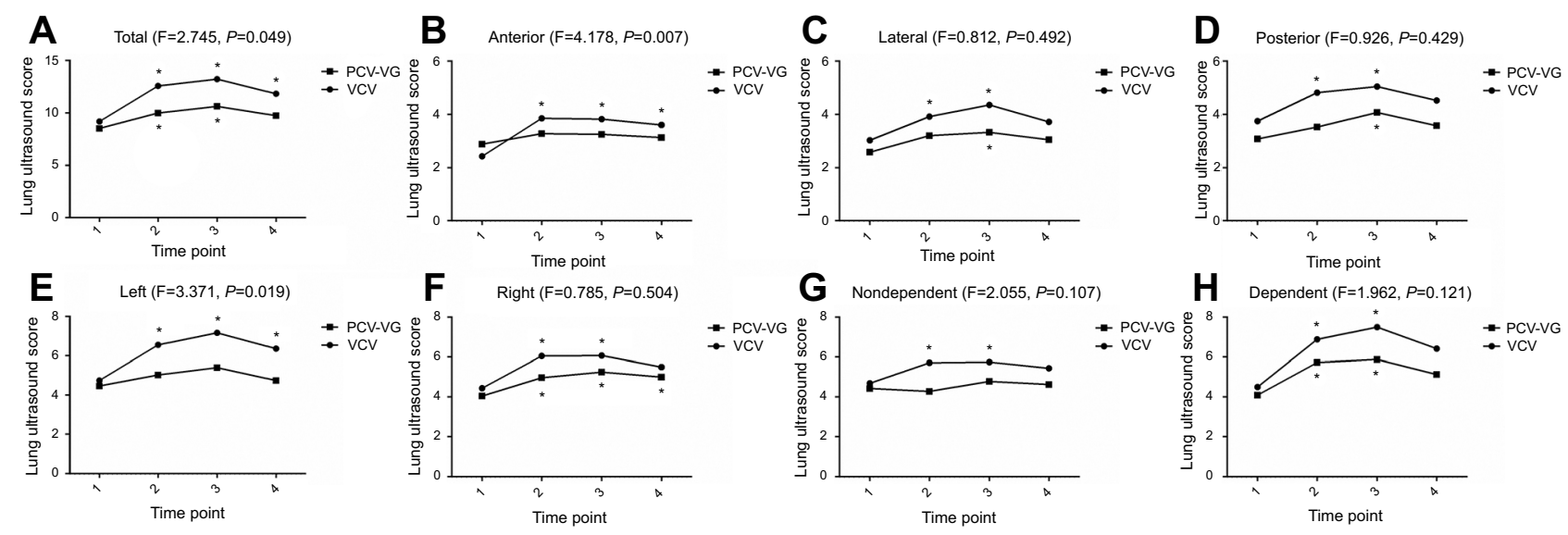

Figure 2 Changes in LUS in the PCV-VG or VCV group.

Note: There were significant effects on LUS observed in the PCV-VG or VCV group (using repeated measures ANOVA). LUS, lung ultrasound score. $* P<0.0083$ (considering Bonferroni correction) compared to the preoperative value.

Moreover, PCV showed reduced stress responses (decrease in cortisol levels and increase in insulin levels) ${ }^{19}$ and even improved right ventricular function. ${ }^{13}$

Considering PCV-VG, the results are similar to $\mathrm{PCV}^{20}$ Unlike a constant flow pattern in VCV, the inspiratory flow pattern in the PCV and PCV-VG is a similar decelerating type with a high initial flow rate. Improved mean airway pressure, ${ }^{21}$ peak inspiratory pressure, and dynamic compliance $^{7}$ and lower IL-8, TNF $\alpha$, and alveolar albumin $^{22}$ suggest a preference for PCV-VG compared to VCV. Additionally, PCV-VG may reduce the risk of hyperventilation or hypoventilation compared to PCV. It is obvious that the tidal volume and minute ventilation changes occurred secondary to the changes in lung compliance and airway resistance under $\mathrm{PCV}^{22}$ Contrary to other studies, one retrospective study ${ }^{23}$ indicated that VCV favored positive results for clinical prognosis. Their study included cases in thoracic or abdominal surgeries, so the discordance was probably due to the volume variance risk of the PCV. To minimize the effect of the changes in lung compliance and airway resistance, we chose orthopedic hip surgery in our study and expected that PCV-VG was not much different from PCV.

Among the several methods to assess pulmonary status, LUS has many advantages. LUS allows dynamic evaluation in clinical situations. In the view of point of care, LUS evaluation is fast and relatively precise. ${ }^{24}$ Zhao et al showed correlations between LUS and several acute respiratory distress syndrome (ARDS) prognostic indices. Their cut-off of LUS for mortality prediction was $16.5 .^{25}$ LUS was closely related to several ARDS prognostic indices (extravascular lung water index, lung injury score, static respiratory system compliance, and
$\mathrm{PaO}_{2} / \mathrm{FiO}_{2}$ ); it could be used to predict risk of death and could serve as a diagnostic marker of ARDS. Early measurement of LUS is a better diagnostic indicator of acute lung injury compared with late measurement. Another study showed correlations between LUS and clinical pulmonary infection score in ventilator-associated pneumonia. ${ }^{26}$ LUS also showed clinical usefulness in sepsis patients with significant correlations regarding the mortality in emergency department sepsis score, the simplified acute physiology score 3 , and respiratory variables.

Moreover, measurement of LUS is noninvasive and safe. Even in extremely preterm neonates with respiratory distress syndrome, LUS showed its usefulness in medical applications. ${ }^{27}$ Besides, LUS are generally economical ${ }^{25}$ although the medical environments vary from country to country.

Early studies of LUS showed good results using the 8region $^{28}$ or 6-region method, based on the simplified lung edema scoring system). ${ }^{29}$ Recent studies indicate that the 12 region method is now being more widely used ${ }^{25}$ because the 12-region method may be more accurate and produce sufficiently reliable results. In cases of non-homogeneous lung pathology, the more regions for LUS evaluation may guarantee the less omission. This was why we used the 12-region method in our study. While early LUS used only longitudinal scans, we used longitudinal and transverse scans in combination at the same lung regions and attained a higher score. The combination of scans was used to compensate for a possible limitation in visualization of the pleura due to decreased intercostal space width. ${ }^{24}$

Our secondary outcome measure was PFT. Because some patients, including those with delirium, were unable 

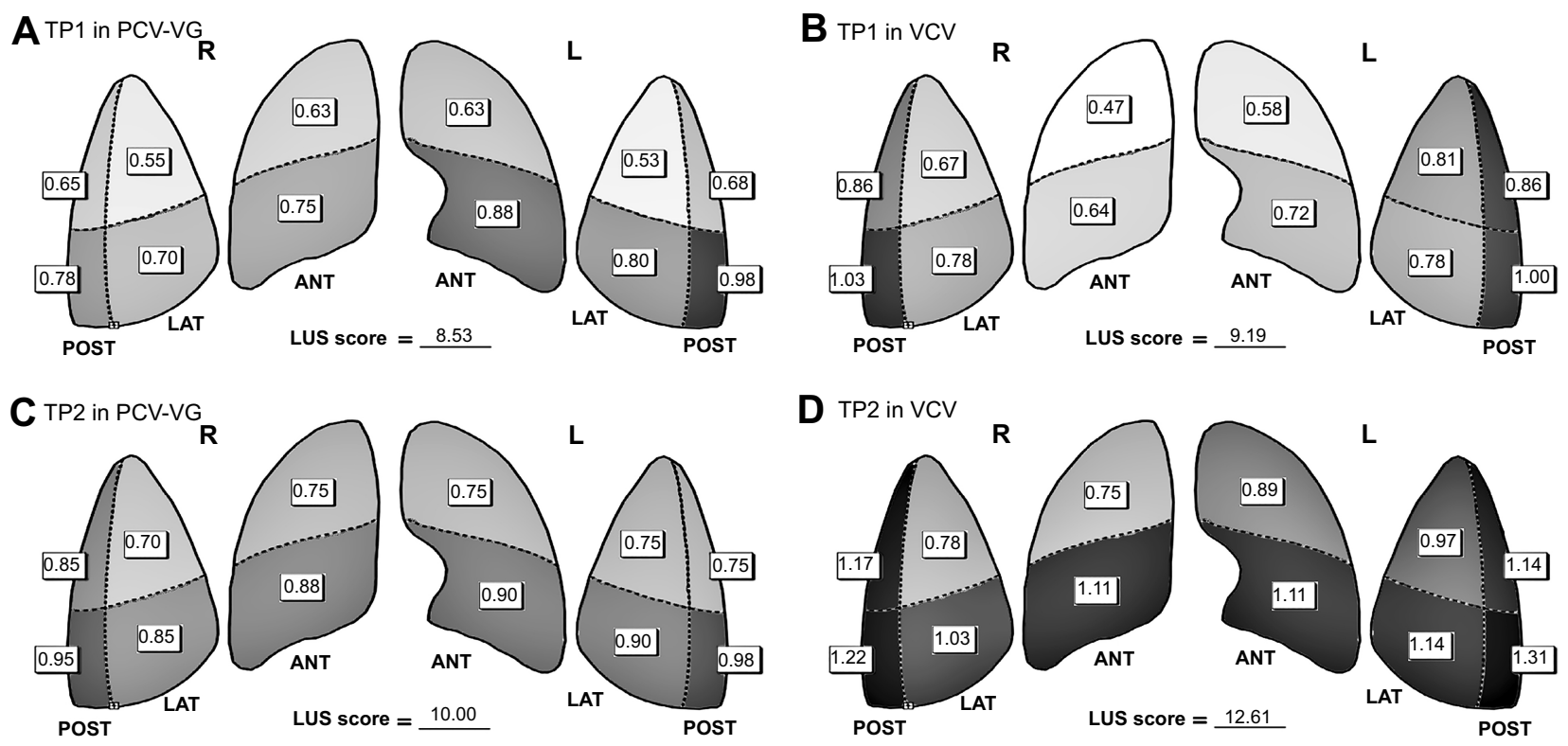

D TP2 in VCV
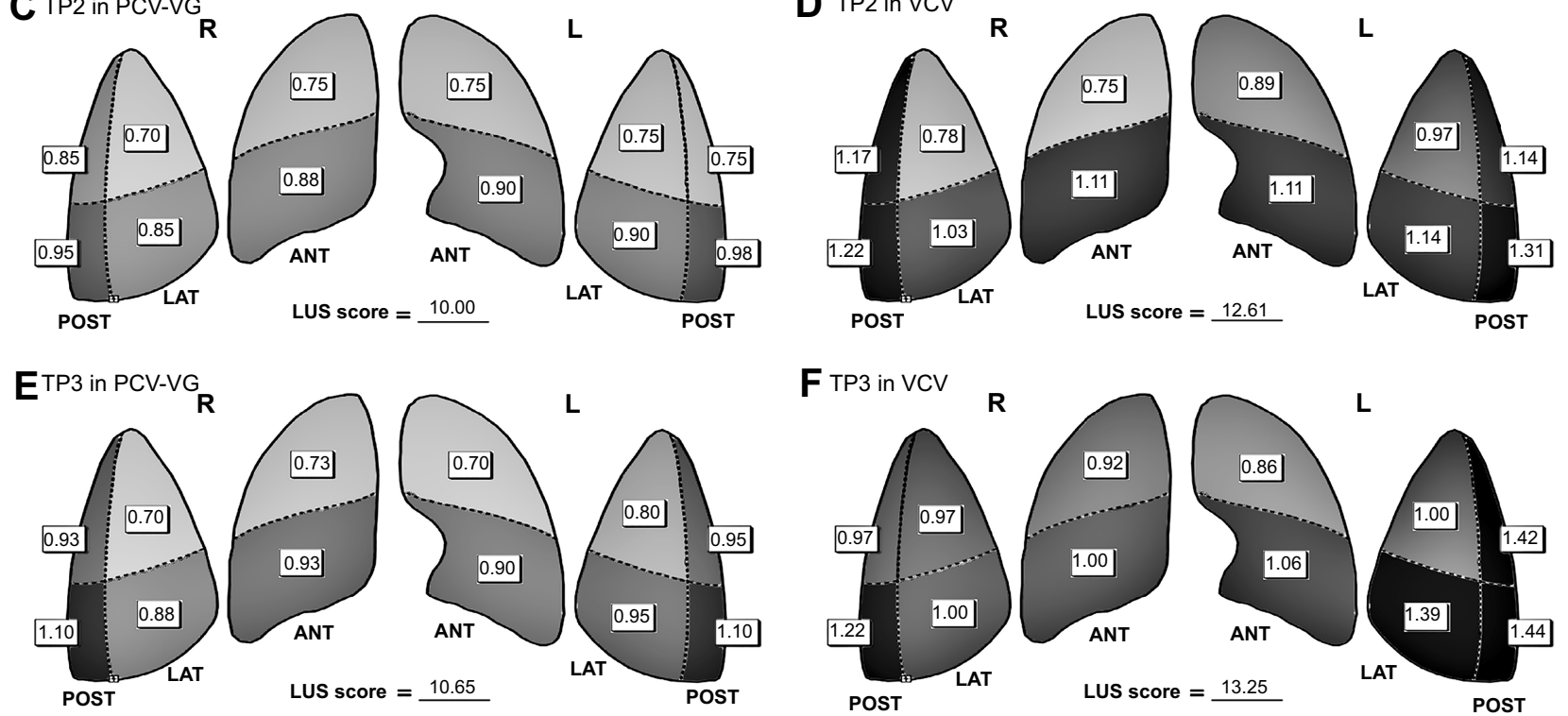

$\boldsymbol{F}_{\mathrm{TP}}$ in VCV
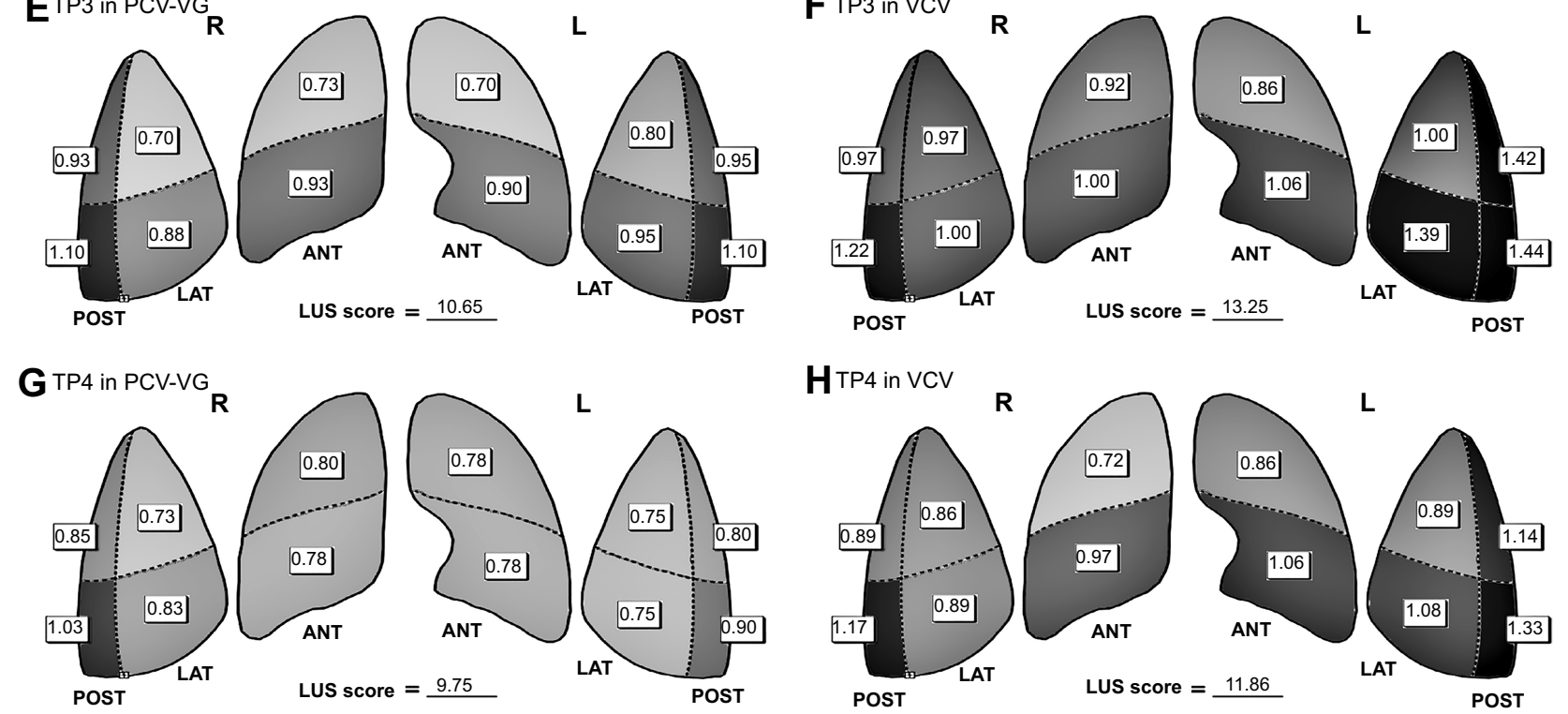

HTP4 in VCV

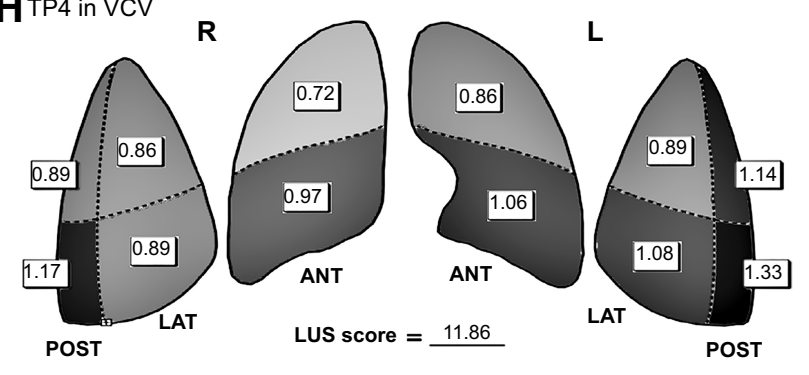

Figure 3 Visual model of LUS results.

Note: An LUS value of 0.5 or less was set to white and a value of 1.5 or more was set to black. Between 0.5 and I.5, when the LUS value was closer to 0.5 , the color was brighter, and when it was closer to I.5, the color was darker. Therefore, a brighter color means a lower LUS, which is preferable.

Abbreviation: LUS, lung ultrasound score.

to cooperate, we analyzed the results of the other patients (86.8\% of all patients). In both groups, fentanyl use and pain scores were comparable in PACU. Although pain scores at the PACU after $1 \mathrm{hr}$ were relatively low, postoperative PFT values did not reach the preoperative level. Unlike the LUS results, PFT did not support the superiority of PCV over VCV.

While planning our study, we made note that other respiratory parameters, including airway pressure or lung compliance, were already reported in other studies and postoperative findings were not different despite significant findings during surgery. The influence of the ventilation mode is liable to be easily overlapped because patient factors or other factors affect patient prognosis much more. ${ }^{5}$ Moreover, there are also compensatory adaptations of each patient to consider. To be more specific, although unlikely in respiratory compromised patients, healthy patients may compensate and overcome perioperative lung problems such as lung edema or atelectasis by themselves. These points seem to be the reason why prior meta- 
analyses have not shown a big difference in comparing PCV-VG and VCV. Elderly patients also show reduced functional reserve and ability to compensate for physiologic stress, even if not as much as compromised patients. ${ }^{30}$ While PCV did not show improved oxygenation in patients with good preoperative pulmonary function, ${ }^{31}$ the advantages of PCV over VCV are seen in the elderly patient with poor pulmonary function. ${ }^{32}$

When changing from the supine to the lateral decubitus position, significant alterations in pulmonary physiology occur. Because pulmonary blood flow is gravity dependent, hydrostatic pressure gradients are established. In the dependent lung, increased interstitial pressure and increased resistance of the extra-alveolar vessels may result in decreased pulmonary blood flow. ${ }^{33}$ Other influences, including vascular factors, make the explanation of physiologic changes more complex. Single-photon emission computed tomographic images of the lung showed a central-to-peripheral gradient in the distribution of blood flow. ${ }^{33}$ Cardiac output and lung volumes also affect the distribution of pulmonary blood flow. Pulmonary vascular resistance is lowest at functional residual capacity of lung volume. Hyperinflation or hypoinflation of the lung may cause increased pulmonary vascular resistance by stretching the capillaries or decreasing the caliber of the extraalveolar Vessels. ${ }^{33}$ In our results, the dependent lung showed greater alteration in LUS than the nondependent lung (Figure 3G and $\mathrm{H}$ ). We expected that PCV-VG would further improve the LUS of the dependent compartment, but the effect was not significant. Rather, the effects were prominent in the left and the anterior compartments (Figure 3B, E). It is difficult to completely explain the mechanisms behind these phenomena, but the anatomical locations of the lung and heart may be influential. These findings also suggest that there is non-homogeneous change in lung aerations based on the ventilation mode. It may support PCV-VG advantages in the old aged patients. Senile emphysema, which is dilated alveola air space, associated with fragmentation of the elastic fibers in alveolar septae, exhibits a homogenous appearance throughout the parenchyma when there is no excessive inflammatory infiltration or destruction of the alveolar septum. ${ }^{34}$ Compared to VCV, PCV-VG seems to provide homogeneous ventilation and better lung aeration in the left and anterior compartments.

Regarding other ventilator settings used in our study, the calculation of the target tidal volume setting was based on predicted body weight rather than actual body weight.
Routine positive end-expiratory pressure (PEEP) of $5 \mathrm{cmH}_{2} \mathrm{O}$ was applied and routine recruit maneuver was not used. Many recent studies ${ }^{35}$ have evaluated various aspects of intervention during intraoperative ventilation, including lower tidal volume based on predicted body weight, higher PEEP, and application of a recruitment maneuver. Their results conflict in some ways, but there is general agreement that improved oxygenation and better compliance are the advantages. However, high PEEP may lead to hypotension and fluid overload. ${ }^{35}$ Using a bagsqueezing technique for recruitment may then lead to lung decruitment when the ventilator is switched back from manual to mechanical ventilation. Other maneuvers, including cycling maneuvers or stepwise tidal volume adjustments, can be inconvenient to clinicians or require a new anesthetic machine. Among the general consensus established in these recent studies, the large clinical trial named PROVHILO ${ }^{36}$ recommended low tidal volume, low PEEP, and no recruitment maneuver as strategies for intraoperative lung-protective ventilation.

Because patient inspiratory effort may produce errors in tidal volume measurement during $\mathrm{PCV}^{37}$ we also maintained constant muscle relaxation during surgery under a TOF guidance. During our study, $2 \mathrm{mg} / \mathrm{kg}$ of sugammadex was sufficient in preventing residual curarization.

This study was not adequately powered to detect different clinical outcomes between the two groups. Several methodological limitations may have resulted in these negative findings. First, our study patients are older but relatively healthy enough to perform PFTs properly. The mean of $\mathrm{PaO}_{2} / \mathrm{FiO}_{2}$ ratios during surgery in our study patients was about $400 \mathrm{mmHg}$, which was far from the ARDS criteria ( $\leq 300 \mathrm{mmHg}$ ). In respiratory-compromised patients, the results of PCV-VG or VCV may show significant differences. Second, there is still a lack of evidence in the non-homogeneous distribution of lung aeration from LUS findings. Moreover, we did not provide any guidance or solution when LUS was assessed as high in the perioperative period.

Despite these shortcomings, this study showed successful application of LUS during the perioperative period. LUS evaluation seemed to be easily applied during surgery. Furthermore, visualization of the LUS model may be the basis for understanding lung physiology and distribution of aeration. The most important factor for postoperative deterioration in lung functions or worse LUS is probably perioperative handling of the lung rather than ventilation mode during surgery. Long term post-operative 
respiratory outcome which was not included in this study may be helpful to clarify the perioperative LUS model. Further study should be required to determine how well LUS reflects lung status and predicts prognosis.

In conclusion, LUS models in the both PCV-VG and VCV groups suggested non-homogeneous and positional change in lung aerations during surgery. In addition to higher dynamic compliance and lower inspiratory peak pressure, PCV-VG showed better LUS results compared to the VCV.

\section{Case data repository}

Authorized individuals with UMIN ID may access to the case data repository. Any download history including downloaded date, UMIN ID and name of the downloader, file name, and affiliation of the downloader will be recorded. The backup and security systems of the UMIN guarantee the long-term storage of data. Please contact to the corresponding author.

Access URL: https://upload.umin.ac.jp/cgi-bin/icdr_e/ ctr_view.cgi?recptno=R000033510.

\section{Acknowledgments}

This report was supported by a Korea University Grant awarded to Dr. Young Ju Won from Korea University (Seoul, South Korea).

\section{Author contributions}

All authors contributed to data analysis, drafting or revising the article, gave final approval of the version to be published, and agree to be accountable for all aspects of the work.

\section{Disclosure}

The authors report no conflicts of interest in this work.

\section{References}

1. Haentjens P, Lamraski G, Boonen S. Costs and consequences of hip fracture occurrence in old age: an economic perspective. Disabil Rehabil. 2005;27(18-19):1129-1141. doi:10.1080/09638280500055529

2. Sprung J, Gajic O, Warner DO. Review article: age related alterations in respiratory function - anesthetic considerations. Can J Anaesth. 2006;53(12):1244-1257. doi:10.1007/bf03021586

3. Zaugg M, Lucchinetti E. Respiratory function in the elderly. Anesthesiol Clin North Am. 2000;18(1):47-58, vi.

4. Bohl DD, Sershon RA, Saltzman BM, Darrith B, Della Valle CJ. Incidence, risk factors, and clinical implications of pneumonia after surgery for geriatric hip fracture. J Arthroplasty. 2018;33(5):15521556.e1551. doi:10.1016/j.arth.2017.11.068

5. Marseu K, Slinger P. Perioperative lung protection. Korean J Anesthesiol. 2017;70(3):239-244. doi:10.4097/kjae.2017.70.3.239
6. Eichenberger A, Proietti S, Wicky S, et al. Morbid obesity and postoperative pulmonary atelectasis: an underestimated problem. Anesth Analg. 2002;95(6):1788-1792. table of contents.

7. Assad OM, El Sayed AA, Khalil MA. Comparison of volume-controlled ventilation and pressure-controlled ventilation volume guaranteed during laparoscopic surgery in Trendelenburg position. J Clin Anesth. 2016;34:55-61. doi:10.1016/j.jclinane.2016.03.053

8. Choi EM, Na S, Choi SH, An J, Rha KH, Oh YJ. Comparison of volume-controlled and pressure-controlled ventilation in steep Trendelenburg position for robot-assisted laparoscopic radical prostatectomy. J Clin Anesth. 2011;23(3):183-188. doi:10.1016/j. jclinane.2010.08.006

9. Lian M, Zhao X, Wang H, Chen L, Li S. Respiratory dynamics and dead space to tidal volume ratio of volume-controlled versus pressure-controlled ventilation during prolonged gynecological laparoscopic surgery. Surg Endosc. 2017;31(9):3605-3613. doi:10.1007/ s00464-016-5392-x

10. Liao CC, Kau YC, Ting PC, Tsai SC, Wang CJ. The effects of volume-controlled and pressure-controlled ventilation on lung mechanics, oxidative stress, and recovery in gynecologic laparoscopic surgery. J Minim Invasive Gynecol. 2016;23(3):410-417. doi:10.1016/j.jmig.2015.12.015

11. Jaju R, Jaju PB, Dubey M, Mohammad S, Bhargava AK. Comparison of volume controlled ventilation and pressure controlled ventilation in patients undergoing robot-assisted pelvic surgeries: an open-label trial. Indian J Anaesth. 2017;61(1):17-23. doi:10.4103/0019-5049.198406

12. Sen O, Umutoglu T, Aydin N, Toptas M, Tutuncu AC, Bakan M. Effects of pressure-controlled and volume-controlled ventilation on respiratory mechanics and systemic stress response during laparoscopic cholecystectomy. Springerplus. 2016;5:298. doi:10.1186/ s40064-016-1963-5

13. Al Shehri AM, El-Tahan MR, Al Metwally R, et al. Right ventricular function during one-lung ventilation: effects of pressure-controlled and volume-controlled ventilation. $J$ Cardiothorac Vasc Anesth. 2014;28(4):880-884. doi:10.1053/j.jvca.2013.09.012

14. Tan J, Song Z, Bian Q, Li P, Gu L. Effects of volume-controlled ventilation vs. pressure-controlled ventilation on respiratory function and inflammatory factors in patients undergoing video-assisted thoracoscopic radical resection of pulmonary carcinoma. $J$ Thorac Dis. 2018;10(3):1483-1489. doi:10.21037/jtd.2018.03.03

15. Mihalj M, Vladić D, Karlović Z, Zadro Ž, Majerić Kogler V. Effects of pressure-controlleda volume-controlled ventilation on hemodynamic and respiratory parameters in patients during laparoscopic cholecystectomy. Acta Clin Croat. 2017;56(3):555-560. doi:10.20471/acc.2017.56.03.24

16. Messeha MM. Effect of switching between pressure-controlled and volume-controlled ventilation on respiratory mechanics and hemodynamics in obese patients during abdominoplasty. Anesth Essays Res. 2017;11(1):88-93. doi:10.4103/0259-1162.186594

17. Movassagi R, Montazer M, Mahmoodpoor A, Fattahi V, Iranpour A, Sanaie S. Comparison of pressure vs. volume controlled ventilation on oxygenation parameters of obese patients undergoing laparoscopic cholecystectomy. Pak J Med Sci. 2017;33(5):1117-1122. doi:10.12669/ pjms.335.13316

18. Hoşten T, Kuş A, Gümüş E, Yavuz Ş, Irkil S, Solak M. Comparison of intraoperative volume and pressure-controlled ventilation modes in patients who undergo open heart surgery. J Clin Monit Comput. 2017;31(1):75-84. doi:10.1007/s10877-016-9824-x

19. Şenay H, Sivaci R, Kokulu S, Koca B, Baki ED, Ela Y. The effect of pressure-controlled ventilation and volume-controlled ventilation in prone position on pulmonary mechanics and inflammatory markers. Inflammation. 2016;39(4):1469-1474. doi:10.1007/s10753-016-0379-2

20. Kothari A, Baskaran D. Pressure-controlled volume guaranteed mode improves respiratory dynamics during laparoscopic cholecystectomy: a comparison with conventional modes. Anesth Essays Res. 2018;12 (1):206-212. doi:10.4103/aer.AER_96_17 
21. Kim MS, Soh S, Kim SY, Song MS, Park JH. Comparisons of pressure-controlled ventilation with volume guarantee and volumecontrolled 1:1 equal ratio ventilation on oxygenation and respiratory mechanics during robot-assisted laparoscopic radical prostatectomy: a randomized-controlled trial. Int J Med Sci. 2018;15(13):1522-1529. doi:10.7150/ijms. 28442

22. Mahmoud K, Ammar A, Kasemy Z. Comparison between pressureregulated volume-controlled and volume-controlled ventilation on oxygenation parameters, airway pressures, and immune modulation during thoracic surgery. J Cardiothorac Vasc Anesth. 2017;31 (5):1760-1766. doi:10.1053/j.jvca.2017.03.026

23. Bagchi A, Rudolph MI, Ng PY, et al. The association of postoperative pulmonary complications in 109,360 patients with pressure-controlled or volume-controlled ventilation. Anaesthesia. 2017;72 (11):1334-1343. doi:10.1111/anae.14039

24. Mongodi S, Bouhemad B, Orlando A, et al. Modified lung ultrasound score for assessing and monitoring pulmonary aeration. Ultraschall Med. 2017;38(5):530-537. doi:10.1055/s-0042-120260

25. Zhao Z, Jiang L, Xi X, et al. Prognostic value of extravascular lung water assessed with lung ultrasound score by chest sonography in patients with acute respiratory distress syndrome. BMC Pulm Med. 2015;15:98. doi:10.1186/s12890-015-0091-2

26. Li LM, Li LH, Guan J, Yang Q, Han JQ, Chao YG. [The value of lung ultrasound score for therapeutic effect assessment of ventilatorassociated pneumonia]. Zhonghua Nei Ke Za Zhi. 2016;55(12):950952. doi:10.3760/cma.j.issn.0578-1426.2016.12.009

27. De Martino L, Yousef N, Ben-Ammar R, Raimondi F, ShankarAguilera S, De Luca D. Lung ultrasound score predicts surfactant need in extremely preterm neonates. Pediatrics. 2018;142(3): e20180463. doi:10.1542/peds.2018-0463

28. Volpicelli G, Elbarbary M, Blaivas M, et al. International evidencebased recommendations for point-of-care lung ultrasound. Intensive Care Med. 2012;38(4):577-591. doi:10.1007/s00134-012-2513-4
29. Santos TM, Franci D, Coutinho CM, et al. A simplified ultrasoundbased edema score to assess lung injury and clinical severity in septic patients. Am J Emerg Med. 2013;31(12):1656-1660. doi:10.1016/j. ajem.2013.08.053

30. Cook DJ, Rooke GA. Priorities in perioperative geriatrics. Anesth Analg. 2003;96(6):1823-1836.

31. Unzueta MC, Casas JI, Moral MV. Pressure-controlled versus volume-controlled ventilation during one-lung ventilation for thoracic surgery. Anesth Analg. 2007:104(5):1029-1033. tables of contents. doi:10.1213/01.ane.0000260313.63893.2f

32. Lin F, Pan L, Huang B, et al. Pressure-controlled versus volumecontrolled ventilation during one-lung ventilation in elderly patients with poor pulmonary function. Ann Thorac Med. 2014;9(4):203-208. doi:10.4103/1817-1737.140125

33. Dunn PF. Physiology of the lateral decubitus position and one-lung ventilation. Int Anesthesiol Clin. 2000;38(1):25-53.

34. Tran D, Rajwani K, Berlin DA. Pulmonary effects of aging. Curr Opin Anaesthesiol. 2018;31(1):19-23. doi:10.1097/aco.000000000 0000546

35. Ball L, Dameri M, Pelosi P. Modes of mechanical ventilation for the operating room. Best Pract Res Clin Anaesthesiol. 2015;29(3):285299. doi:10.1016/j.bpa.2015.08.003

36. Hemmes SN, Gama de Abreu M, Pelosi P, Schultz MJ, PROVE Network Investigators for the Clinical Trial Network of the European Society of Anaesthesiology. High versus low positive end-expiratory pressure during general anaesthesia for open abdominal surgery (PROVHILO trial): a multicentre randomised controlled trial. Lancet. 2014;384(9942):495-503. doi:10.1016/s0140-6736(14) 60416-5

37. Chatburn RL, Mireles-Cabodevila E, Sasidhar M. Tidal volume measurement error in pressure control modes of mechanical ventilation: a model study. Comput Biol Med. 2016;75:235-242. doi:10.1016/j. compbiomed.2016.06.011
Clinical Interventions in Aging

\section{Publish your work in this journal}

Clinical Interventions in Aging is an international, peer-reviewed journal focusing on evidence-based reports on the value or lack thereof of treatments intended to prevent or delay the onset of maladaptive correlates of aging in human beings. This journal is indexed on PubMed Central, MedLine, CAS, Scopus and the Elsevier
Bibliographic databases. The manuscript management system is completely online and includes a very quick and fair peer-review system, which is all easy to use. Visit http://www.dovepress.com/ testimonials.php to read real quotes from published authors. 